\title{
LA INNOVACIÓN DESDE LA PERSPECTIVA DEL CONOCIMIENTO Y COMPETITIVIDAD DE LAS EMPRESAS MANUFACTURERAS DE LA REGIÓN CARIBE
}

\author{
Arcesio Rodrigo López Pérez ${ }^{1}$
}

Recibido: 27 de abril de 2013

Aceptado: 12 de agosto de 2013

\section{Resumen}

Desde la perspectiva del conocimiento y competitividad de las empresas manufactureras de la Región Caribe, se está dando un análisis a partir de una definición de innovación estratégica, desde el punto de vista del conocimiento, la relación de este con la acción y sus implicaciones desde la perspectiva de la competitividad.

Dicho análisis se realizó en los meses de enero a marzo en las empresas manufactureras de la Región Caribe, a través de una metodología cualitativa- explicativa, a través de un ejercicio investigativo, cuyos resultados serán explicados a lo largo del texto, y se podrá visualizar dentro de un contexto general del conocimiento estratégico y la aplicabilidad que las empresas de la Región Caribe tienen como ventajas competitivas y comparativas en la innovación de productos y servicios.

Los resultados obtenidos, arrojaron que las empresas del sector manufacturero, integran de manera estratégica el conocimiento con la innovación en el diseño y desarrollo de productos, buscando generar las estrategias que permitan satisfacer las necesidades de los clientes.

Palabras clave: innovación, competitividad, desarrollo de productos, ventajas, estrategias

\footnotetext{
Administrador de Empresas con énfasis en Alta Gerencia de la Universidad Simón Bolívar, Especialista en seguridad Penitenciaria de la Escuela Penitenciaria Nacional Enrique Low Murtra. Especialista en Pedagogía para el Desarrollo del Aprendizaje Autónomo de la Universidad Nacional Abierta y a Distancia. Candidato a Maestría en Administración de las Organizaciones UNAD.
} 


\section{INNOVATION FROM THE PERSPECTIVE OF KNOWLEDGE AND COMPETITIVENESS OF COMPANIES MANUFACTURING OF THE CARIBBEAN REGION}

\section{Abstract}

From the knowledge and competitiveness perspective of Manufacturing companies in the Caribbean Region, an analysis is being performed, starting with a definition of strategic innovation -from the knowledge viewpoint - its relation to action and its implications from the competitiveness perspective.

The analysis was carried out throughout September and October, in the manufacturing companies of the Caribbean Region. And a quantitative and explicative methodology was used. performed a research project, which results will be explained across the text. And it could be visualized within a general context of the strategic knowledge and the applicability that the companies in the Caribbean Region have as competitive and comparative advantages in the innovation of products and services.

The results obtained from the research showed that the companies in the manufacturing sector incorporate strategically the knowledge into the design and development of products, in order to generate strategies that allow satisfying the customer's needs

Keywords: Innovation, Competitiveness, Development of Products, Advantages, Strategies.

\section{Introducción}

Esta es una época de constantes cambios donde los mercados, los productos y los competidores se transforman tan rápido que las organizaciones difícilmente responden a las nuevas circunstancias; razón por la cual se vuelve muy complejo mantener un nivel de competitividad lo suficientemente sólido y sostenible como para garantizar una posición representativa en el mercado.

Las Pequeñas y Medianas Empresas (PYMES) colombianas no son ajenas a esta realidad, toda vez, que representan un alto porcentaje en la industria, ya sea en generación de empleo como en cantidad de empresas, sus propietarios se caracterizan por ser innovadores, creativos, tienen gran adaptabilidad y desarrollan estrategias comerciales que les permiten adaptarse a las condiciones cambiantes que se presentan en el mercado global, el cual es muy exigente en términos de la calidad de los productos y en la eficiencia de los servicios ofertados.

Por su parte, las ventajas competitivas en el sector de las empresas manufactureras, cobran gran importancia, debido al desarrollo de nuevos productos gustos y preferencias del cliente, especialmente el empresario debe apuntar su estrategia diferenciadora a través de la ventaja única, tal como lo afirma Porter (2010: 33) en su propuesta de valor único. Apuntó varias preguntas que es necesario plantearse antes de elaborar una estrategia de valor único: ¿A qué clientes queremos dirigirnos?, ¿Qué necesidades especificas queremos cubrir?, ¿Qué precio le pondremos al producto?, esa propuesta además, debe ser diferente de la de nuestro competidor, y para ello hay que valerse de todos los aspectos de la cadena de valor (materiales, producción, ventas, distribución) 
con el único propósito de ser diferentes. La competitividad en las empresas es uno de los factores intangibles que plantea más dificultad en su gestión. El nuevo escenario se identifica con la aceleración del cambio tecnológico y el acortamiento del ciclo de la vida de los productos, de ahí la importancia estratégica de realizar una eficaz gestión de la tecnología en la empresa.

Una empresa alcanza ventaja competitiva cuando obtiene resultados superiores (Porter, 1980); es decir, cuando la utilidad excede el costo de oportunidad de los recursos utilizados en la elaboración del bien o servicio.

Una vez definida la estrategia de valor único, es fundamental aportarle a la administración, donde se hace necesario para el desarrollo de la empresa en este caso para auto-organizarse, la capacidad de interacción entre las partes de la empresa y sus procesos emergentes de conducta coherente.

De esta manera la estrategia para el Desarrollo del Producto pretende incrementar las ventas mediante una modificación y mejoría de los productos o servicios. Por regla general, para el desarrollo del producto se requiere destinar buen presupuesto económico para investigación y desarrollo, que permita dinamizar y garantizar resultados tangibles.

Morin (1988) propone analizar la complejidad desde la teoría de los sistemas, que lo simple no existe, sólo lo simplificado, de esta manera la ciencia moderna ha construido situaciones experimentales no complejas. Es por esto que la innovación es sin duda una de las características más importantes e interesantes de las pequeñas y medianas empresas, no solo está basada en lo tecnológico sino también en el producto que se comercializa, en los procesos de fabricación organizadamente a través de la creatividad y la capacidad de crear que mejoren el desempeño de los empleados a través del marketing.
Para Schumpeter (1934) considera la innovación, como la introducción en el mercado de un nuevo bien o una nueva clases de bienes, el uso de nuevas fuentes de materias primas y la incorporación de nuevos métodos de producción.

Según Freeman (1974), la innovación es el proceso de integración de la tecnología y los inventos para crear o mejorar un producto, un proceso o un sistema.

Para Drucker (1988) la innovación se ha convertido en un término clave para los gerentes de las empresas que hacen parte de la sociedad del conocimiento y la información, muchos pensadores coinciden en el concepto de cambio estratégico en sus procesos horizontales y verticales.

Porter (1991) menciona que el concepto significativo de cualquier empresa es la competitividad y desarrollo de productos ${ }^{1}$ a través de su crecimiento, el cual está estrictamente relacionado con la productividad en términos de calidad total en sus productos y servicios, pues se dice que una compañía tiene Ventaja Competitiva cuando cuenta con una mejor posición que los rivales para asegurar a los clientes y defenderse contra las fuerzas competitivas.

En este sentido existe en el contexto empresarial muchas fuentes de ventajas competitivas, como la elaboración del producto con la más alta calidad, el proporcionar un servicio diferenciador a los clientes, lograr menores costos en los rivales, tener una mejor ubicación geográfica, diseñar un producto que tenga un mejor rendimiento que las marcas de la competencia. No obstante, las Pymes representan para nuestro país una gran importancia social para el desarrollo económico impactando directamente en la generación de empleos, y desarrollo de mercados beneficiando a las personas de bajo estrato.

Desarrollo entendido como el valor agregado en la diferenciación del bien o servicio. 


\section{Desarrollo}

La innovación desde el análisis de los procesos de desarrollo económico permite evaluar su eficacia y reflexionar sobre la aplicabilidad de los procesos de desarrollo social en la construcción de indicadores de innovación útiles, Las Pequeñas y Medianas Empresas (PYMES) colombianas representan un alto porcentaje en la industria, ya sea en generación de empleo como en cantidad de empresas, y requieren adaptarse a las condiciones cambiantes que se presentan en el mercado global, el cual es muy exigente en términos de la calidad, es por ello que las ventajas competitivas en el sector de las empresas manufactureras, cobran gran importancia, en un sentido amplio, afirma Godin (2008), la innovación se ha convertido en un lema de la sociedad del mercado inconmensurable y en el filtro para resolver muchas falencias dentro de la gerencia (Godin, 2008).

En términos más específicos, el proceso de innovación consiste mejorar la posición competitiva de las empresas permitiendo la capacidad de asumir los cambios y ampliar capacidades creativas, la búsqueda de mejores condiciones competitivas por parte de las empresas $y$, en general, la aproximación de la investigación básica y la tecnología como fenómeno portador de transformaciones de la sociedad del conocimiento, a través de como una ruptura intencional del equilibrio empresarial.

Para Schumpeter la innovación, es prestar atención en la búsqueda de nuevos marcos conceptuales que permitieran el sentido muy preciso de dinamizar la dimensión competitiva de la economía capitalista. Este concepto forja a la empresa como un asunto social e interactivo en el cuadro de un entorno social específico y sistémico.

Medir innovación en la forma en que actualmente se hace ¿da cuenta de la realidad social en las empresas regionales? ¿Es posible generar el esfuerzo innovador en las «tecnologías sociales»?, hoy la tarea de incorporar la sociedad a los indicadores de innovación se hace importante desde el concepto de gestión empresarial del conocimiento. Implicando con esto el borrar del pensamiento que las fronteras del conocimiento se encuentran en cada estructura mental.

En este sentido existe en el contexto económico una serie de ventajas competitivas, como la elaboración de productos con la más alta calidad, proporcionar un servicio con valor agregado. Las Pymes representan para nuestro país una gran importancia social para el desarrollo económico impactando directamente en la generación de empleos, y desarrollo de mercados beneficiando a las personas de bajo estrato. Por ello con le desarrollo de este articulo de investigación se analizaran los contextos fácticos de la innovación en las empresas manufactureras.

\section{Metodología}

El diseño del artículo se llevó a cabo a través de una investigación cualitativa explicativa, el universo del estudio estuvo constituido por los establecimientos que funcionan en la Región Caribe de acuerdo con la clasificación Industrial. La población objetivo fueron los establecimientos industriales que funcionan en la Región Caribe.

El diseño longitudinal, en el desarrollo del artículo está constituido por todos los establecimientos industriales que funcionan en la región Caribe. El muestreo utilizado fue de tipo probabilística, más exactamente, un muestreo aleatorio estratificado de empresas, pues se estableció como supuesto para la construcción de los 22 grupos, que en cada uno de ellos se agrupan empresas homogéneas. Esta estratificación se realizó buscando reducir al mínimo la variabilidad debida al azar.

El cálculo del tamaño de la muestra tuvo en cuenta los siguientes parámetros:

Una probabilidad de ocurrencia $(P)$ de 0,8 ; una confiabilidad del $95 \%$. Desde estos parámetros 
el tamaño inicial de la muestra fue de 1851 empresas; sin embargo, esperando una tasa de no respuesta alrededor del $20 \%$, se consideró un tamaño de muestra de 112 encuestas en las empresas, sin embargo en la realidad fáctica se aplicaron 82 encuestas.

Con el propósito de obtener una buena representatividad, se hizo la fijación de la muestra en los 22 grupos creados, mediante simulaciones que buscaban que el error estándar relativo en cada uno de los grupos fuera similar al $20 \%$ inicialmente establecido. Estas simulaciones se hicieron variando la probabilidad de ocurrencia entre 0,8 o más. La fórmula utilizada para el cálculo del tamaño de la muestra por grupo fue:

$$
n_{i}=\frac{N P Q}{N(E S r e l \times P)^{2}+P Q}
$$

Donde:

$\mathrm{N}=$ Tamaño del universo de estudio.

$\mathrm{P}=$ Probabilidad de ocurrencia del fenómeno (para el estudio se decidió tomar como probabilidad de ocurrencia 0,6).

$Q=1-P$

ESrel = Error estándar relativo de la estimación, del $20 \%$.

Para la selección de la muestra se utilizó un muestreo aleatorio simple.

\section{Resultados}

Con el presente artículo se pretende presentar el panorama de la innovación en la Región Caribe, desde el enfoque de Gestión del conocimiento, la utilización de las TIC desde la perspectiva de la competitividad y la Innovación estratégica, en las empresas manufactureras.

\begin{tabular}{|c|c|c|c|}
\hline No. & Sector & Universo & Muestra encuestada \\
\hline 1 & Alimentos & 256 & 6 \\
\hline 2 & Bebidas, tabaco & 22 & 4 \\
\hline 3 & Lácteos & 21 & 4 \\
\hline 4 & Textiles & 102 & 5 \\
\hline 5 & Confecciones & 85 & 5 \\
\hline 6 & Cuero, calzado, marroquinería & 162 & 6 \\
\hline 7 & Madera & 12 & 4 \\
\hline 8 & Papel y cartón & 54 & 5 \\
\hline 9 & Edición, impresión & 121 & 6 \\
\hline 10 & Coque, petróleo, caucho & 12 & 4 \\
\hline 11 & Química & 27 & 5 \\
\hline 12 & Otros químicos & 145 & 6 \\
\hline 13 & Plástico & 142 & 6 \\
\hline 14 & Mineral no metálico y vidrio & 25 & 4 \\
\hline 15 & Metálica básica & 41 & 5 \\
\hline 16 & Productos metálicos & 148 & 6 \\
\hline 17 & Maquinaria & 157 & 6 \\
\hline 18 & Equipo eléctrico & 46 & 5 \\
\hline 19 & Eq. precisión, radio, TV & 22 & 4 \\
\hline 20 & Autopartes & 74 & 5 \\
\hline 21 & Muebles & 123 & 6 \\
\hline \multirow[t]{2}{*}{22} & Otras industrias & 54 & 5 \\
\hline & Total & 1851 & 112 \\
\hline
\end{tabular}

Fuente: Dane - 2012 -sector de las economías 
Tamaño muestra: Análisis propio aplicación formula. Para tal efecto realizaremos un total de 112 encuestas con los siguientes enfoques:

- Prácticas de gestión del conocimiento

- El diseño y el desarrollo de nuevos productos

Al analizar la distribución de la muestra de acuerdo con el tamaño de la empresa, definido según el número de empleados, la encuesta aplicada tiene el enfoquede gestión del conocimiento, la utilización de las TIC desde la perspectiva de la competitividad estratégica, cuyo objetivo es identificar las características relevantes para las empresas y en el enfoque de innovación estratégica.

Como resultado se aplicaron 82 encuestas a empresas del sector manufacturero representadas así:

\section{Prácticas de gestión del conocimiento}

\begin{tabular}{|c|c|c|c|}
\hline Practica & Nula & Media & Alta \\
\hline Adquisición del conocimiento & 27 & 40 & 15 \\
\hline Impulsa la cooperación con otras empresas. & 45 & 25 & 11 \\
\hline \multirow[t]{2}{*}{ Se motiva a los empleados a participar en redes } & 9 & 54 & 18 \\
\hline & Nula & Media & Alta \\
\hline Conversión del conocimiento & 13 & 24 & 45 \\
\hline Informar a los empleados sobre las últimas innovaciones & 19 & 41 & 21 \\
\hline Existe la participación estratégica & 5 & 14 & 62 \\
\hline Garantizar mejores prácticas en la organización & 15 & 28 & 38 \\
\hline \multirow[t]{2}{*}{ Los empleados comparten conocimientos y experiencias } & 12 & 11 & 58 \\
\hline & Nula & Media & Alta \\
\hline Aplicación del conocimiento & 17 & 24 & 40 \\
\hline La empresa cuenta con bases de datos actualizadas de sus clientes & 2 & 12 & 67 \\
\hline La empresa tiene directorios o listas de correo de expertos & 46 & 24 & 11 \\
\hline Hay acceso a bases de datos actualizadas de la organización & 14 & 26 & 41 \\
\hline Los empleados tienen acceso datos de la empresa & 5 & 35 & 41 \\
\hline Total & & & \\
\hline
\end{tabular}

\section{El diseño y el desarrollo de nuevos productos}

\begin{tabular}{llll} 
& \multicolumn{1}{c}{ Pula } & Media & Alta \\
Generación de la idea & $\mathbf{2}$ & $\mathbf{1 4}$ & $\mathbf{6 5}$ \\
Generación del nuevo conocimiento requerido & 5 & 22 & 54 \\
Estrategia de los usos y funciones del producto & 2 & 26 & 53 \\
Visualización de las formas y estética del producto & 1 & 35 & 45 \\
Definición de especificaciones técnicas & 12 & 25 & 44 \\
Desarrollo y realización de pruebas & 2 & 34 & 45 \\
Desarrollo del empaque y/o embalaje & 2 & 26 & 53 \\
Desarrollo de nuevos modos de interacción cliente-producto, & 1 & 33 & 47 \\
Desarrollo de la imagen y/o publicidad & 2 & 22 & 57 \\
\hline
\end{tabular}


Prácticas de gestión del conocimiento

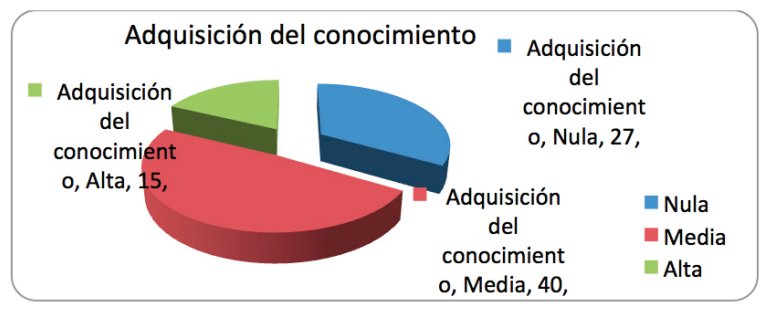

Figura 1. Resultado encuesta

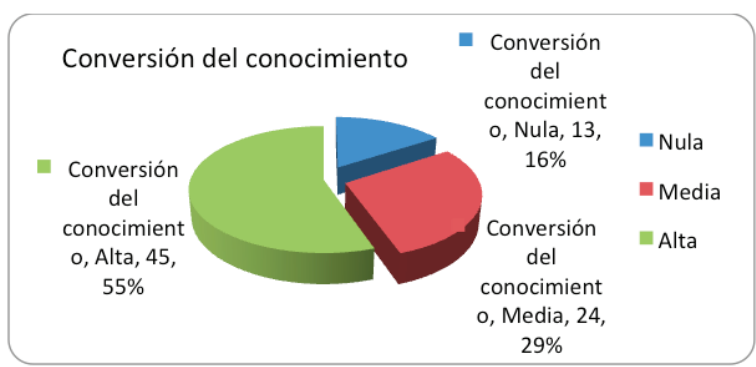

Figura 2. Resultado encuesta

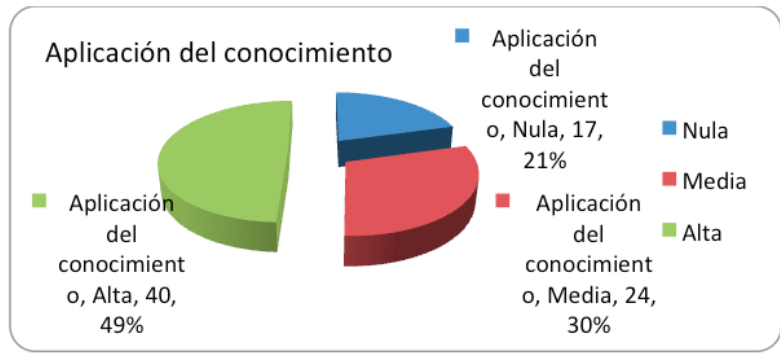

Figura 3. Resultado encuesta

Como análisis en las prácticas del conocimiento las empresas de este sector el $82.71 \%$ genera prácticas de gestión de conocimiento para sus empresas con la fuerza productiva, notándose un considerable ascenso de la conversión del conocimiento como actor estratégico en los proceso internos.

\section{El diseño y el desarrollo de nuevos productos}

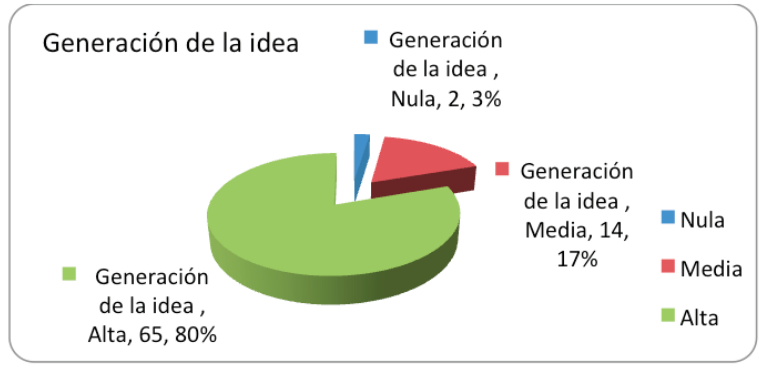

Figura 4. Resultado encuesta

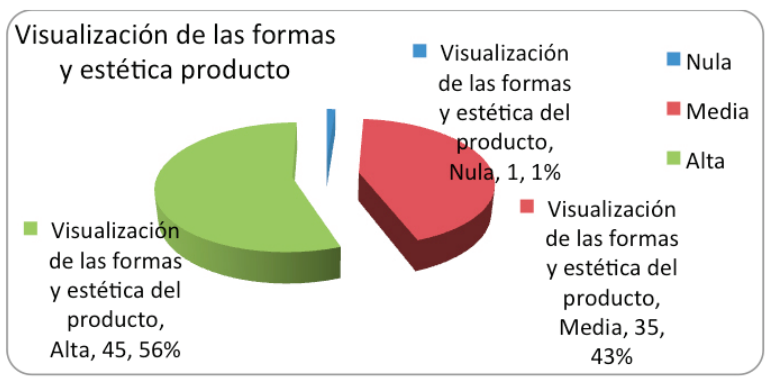

Figura 5. Resultado encuesta

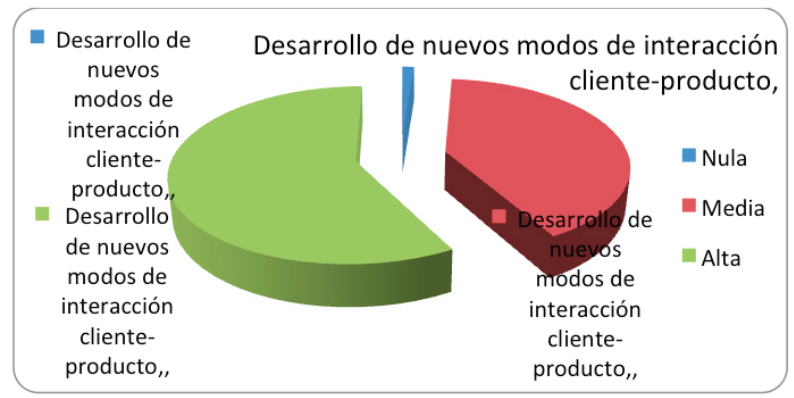

Figura 6. Resultado encuesta

Integración diseño y desarrollo de nuevos productos

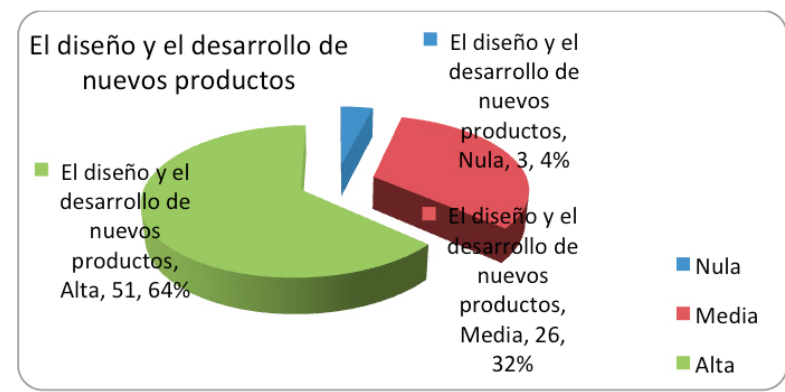

Figura 7. Resultado encuesta 
La medición arrojo que el $63.51 \%$, de las empresas del sector manufacturero de la Costa Atlántica, una vez generan la gestión en el proceso del conocimiento, integran de manera estratégica el conocimiento con la innovación en dos enfoques «Diseño y Desarrollo» de nuevos productos. Notándose un fuerte impacto en la generación de ideas en un porcentaje e del orden de los $80.24 \%$.

\section{Discusión}

La definición del concepto de innovación estratégica, la tomamos como referencia de los autores señalados como Schumpeter, Freeman, Peter Drucker, Medina Salgado y Espinoza Espíndola, abordando los conceptos de ventajas, competitividad, desarrollo de productos, estrategias e innovación. ¿Por qué estudiar la variable innovación? Tal parece que esta variable desliga una serie de procesos al interior de las organizaciones industriales modernas, y estos a su vez transforman y son transformados por sus correlatos contextuales.

Desde Michael Porter, el resultado obtenido en la gestión de la conversión del conocimiento, es garantizar mejores prácticas en la organización el $85.28 \%$; las empresas manufactureras de la región Caribe aplican las ventajas competitivas a través de la gestión del conocimiento. (Ver figura 2 conversión del conocimiento). Ciertos académicos norteamericanos se han interesado por estas limitantes, por ejemplo Abernathy y sus asociados (ABERNATHY y otros, 1983), manejan en sus estudios el concepto de demaduración, para escoger la posibilidad de cambiar la dinámica industrial y trasladarla a un punto en donde los productos provenientes de la tecnología y la innovación, se conviertan nuevamente en herramientas generadoras de ventajas competitivas. Un elemento fundamental en el proceso de «de-maduración» se relaciona con el mejoramiento competitivo en el área de operaciones y de administración de la produc- ción. Hayes y Wheel Wright (1984) últimamente han sugerido que este proceso demandará en muchos casos la total integración de las atenciones relacionadas con las operaciones, la producción y los altos niveles de la firma y en resultado con la administración estratégica.

En su complejidad, el resultado del desarrollo de nuevos modos de interacción cliente-producto, es del $98.76 \%$ en la empresas manufactureras de la Región Caribe, es decir existe una dinámica desde la teoría de los sistemas en la complejidad como lo propone Edgar Morín. (Ver figura 6 Modos de Interacción).

La Integración diseño y desarrollo de nuevos productos, está inmersa dentro de las estrategias de los gerentes de las empresas manufactureras de la región Caribe, ya quela medición arrojo que el $63.51 \%$, de las empresas del sector manufacturero, una vez generada la gestión; integran de manera estratégica el conocimiento con la innovación en dos enfoques «Diseño y Desarrollo» de nuevos productos (Ver figura 7 diseño y desarrollo).

La innovación busca generar la estrategia para satisfacer un deseo o una necesidad de manera integral al cliente como parte interesada, toda vez que en las prácticas del conocimiento las empresas de este sector el $82.71 \%$ generan prácticas de gestión de conocimiento para sus empresas con la fuerza productiva, razón por la cuallas empresas manufactureras deben apuntarle desde la perspectiva de la competitividad, la óptima utilización de las TIC desde la innovación estratégica.

La innovación es la herramienta definida del empresariado innovador. Para ello, la innovación consiste en la acción de otorgar a los recursos con una nueva capacidad de producir riqueza. La innovación crea un recurso. Cualquier cambio en el potencial productor de riqueza ya existente, también es innovación. 
La innovación no tiene que ser meramente técnica ni siquiera tiene que ser una «cosa». Pocas innovaciones técnicas pueden disputar con innovaciones sociales como los periódicos o los seguros. En cuanto se implanta una innovación; cambia la economía, de dirigirse por el proveedor a ser dirigida por el comprador.

La administración de las empresas, tiene como vital fundamento «el conocimiento útil» que concede al hombre la capacidad para impulsar a las personas, aunque posean un diferente potencial y diferentes grados de conocimiento, mediante el trabajo organizacional, esta es la innovación de este siglo.

La peculiaridad «innovadora» no se aplica exclusivamente a las entidades económicas. En opinión de Peter Drucker, no podría hallarse un mejor ejemplo para la Historia de las empresas innovadoras que la creación y desarrollo de las universidades modernas (Drucker, 1989).

\section{Referencias}

Barney, Jay B. (1991). Firm resources and sustained competitive advantage. Journal of Management, 17: 99-120.

Caldart, A. y Ricart, J. (2004). Corporate Strategy Revisited: A View from Complexity Theory. EMR, 1 (1): 96-104

Cardona, Raúl A. (2010). Planificación financiera en las pymes exportadoras. Caso de Antioquia, Colombia. Revista Administer, Universidad EAFIT, Medellín, Colombia. 16: 50-74.

Connor y Prahalad 1996. La participación de tecnologías de la información, I technnol J., 9: 1504-1516
Departamento Administrativo Nacional de Estadística - DANE (2011 -2012). Cuarta Encuesta de desarrollo e innovación tecnológica en la industria manufacturera EDIT IV. Bogotá: DANE.

Drucker, P. (1993). La sociedad post-capitalista. Bogotá: Norma.

Drucker, P. F. (1988). La innovación y el empresariado innovador: la práctica y los principios. México: Hermes.

Freeman, Christopher (1974). La teoría económica de la innovación industrial. Madrid: Editorial Alianza Universidad.

Langebaek, A. y D. Vásquez (2007). Determinantes de la actividad innovadora en la Industria manufacturera colombiana. Borradores de Economía del Banco de la República de Colombia №433.

Peteraf, Margaret. (1993). The Cornestones of Competitive Advantage: A Resource - Base View. In Strategic Management Journal, 3: 179-191.

Porter, Michael. (1991). Towards a Dynamic Theory of Strategy. In Strategic Management Journal, 12: 95-117.

Porter, M. (1980). Competitive Strategy: Techniques for Analyzing Industries and Competitors. New York: the Free Press.

Porter, M. (1985). Competitive Advantage: Creating and Sustaining Superior Performance. New York: the Free Press.

Porter, M. (1996). What is Strategy? Harvard Business Review November-December: 61-78.

Schumpeter, J. (1934). The Theory of Economic Development, Cambridge, Mass: Harvard University Press.

Godin, B. (2008). Innovation: the History of a Category, paper no. 1, Project on the Intellectual History of Innovation, Montreal, INRS. 